\title{
Revisão Sistemática da Literatura: Formação de Grupos na Aprendizagem Colaborativa com Suporte Computacional
}

\author{
Leonam C. Oliveira ${ }^{1}$, Selma S. Rosa ${ }^{2}$, Andrey R. Pimentel ${ }^{1}$ \\ ${ }^{1}$ Departamento de Informática - Universidade Federal do Paraná (UFPR) \\ Curitiba - PR - Brasil \\ ${ }^{2}$ Campus Avançado de Jandaia do Sul - Universidade Federal do Paraná (UFPR) \\ Jandaia do Sul - PR - Brasil \\ leonamcdeoliveira@gmail.com, selmasantos@ufpr.br, andrey@inf.ufpr.br
}

\begin{abstract}
Computer-Supported Collaborative Learning aims to help in the process of teaching and learning based on social interaction between students. Several algorithms and techniques are applied in the formation of groups for collaborative learning, as well as in the use of characteristics and attributes of students to carry out this training. This research aimed to perform a systematic review of the literature to update the information regarding the formation of the groups and also a comparison of the results with other previously performed reviews. The extracted data highlight the divergences of data extracted between the reviews and the large number of solutions proposed in the area research.
\end{abstract}

Resumo. A aprendizagem colaborativa com suporte computacional auxilia no processo de ensino e de aprendizagem a partir da interação social entre estudantes. Diversos algoritmos e técnicas são aplicados na formação dos grupos para a aprendizagem colaborativa, assim como na utilização de características e atributos dos estudantes para realizar esta formação. Esta pesquisa objetivou realizar uma revisão sistemática da literatura para a atualização das informações quanto à formação dos grupos e também uma comparação dos resultados com outras revisões previamente realizadas. Os dados extraídos salientam as divergências de dados extraídos entre as revisões e a grande quantidade de soluções propostas nas pesquisas da área.

\section{Introdução}

A aprendizagem colaborativa com suporte computacional, em inglês computersupported collaborative learning (CSCL), objetiva o desenvolvimento de softwares que propiciem a aprendizagem em grupo, oferecendo atividades criativas de exploração intelectual e interação social, atuando como mediação entre os membros do grupo [Stahl et al., 2006]. Entre as vantagens identificadas por estudantes na utilização CSCL, há o ganho de conhecimento, aquisição de habilidades, melhora na interação social e na realização de tarefas em grupo, contribuindo para o processo de ensino e de aprendizagem [Chen et al., 2018].

A formação dos grupos é um entre os processos para a efetiva aplicação da CSCL no contexto ao qual se propõe. Existem algumas abordagens para formar grupos: 
aleatória, seleção pelos próprios estudantes, seleção pelo docente e a possibilidade de utilizar diferentes características dos estudantes para a formação dos grupos [Costaguta, 2015]. Sendo que, na literatura, há diversas técnicas e algoritmos para que essa formação ocorra [Cruz e Isotani, 2014]. Pesquisas recentes, como de [Reis et al., 2018], sugerem como desafio o estudo de características dos estudantes, neste caso estados afetivos, para a aplicação na formação de grupos para a aprendizagem colaborativa.

Diversas revisões sistemáticas da literatura (RSL) são desenvolvidas objetivando compreender e melhorar o processo de formação dos grupos [Citadin et al., 2014; Maqtary et al., 2017] e também questões específicas como: quais são os algoritmos e técnicas utilizadas [Cruz e Isotani, 2014] e quais critérios são utilizados na formação [Amara et al., 2016]. Este estudo busca realizar uma RSL, atualizando as informações relacionadas aos processos da formação de grupo na CSCL, assim como a comparação dos resultados obtidos com os dados extraídos pelas outras RSL.

\section{Metodologia}

A RSL é um método científico para identificação, seleção, coleta e análise de dados a partir de um processo rigoroso e explícito [Farenhof e Fernandez, 2016], sendo importante na literatura da área para compreender os processos da formação de grupos na aprendizagem colaborativa com suporte computacional. As etapas da revisão foram baseadas, com adaptações, no estudo proposto por [Conforto et al., 2011], que sugerem um roteiro chamado de RBS Roadmap. A Figura 1 traz uma visão geral sobre as etapas necessárias para a realização da revisão, segundo [Conforto et al., 2011].
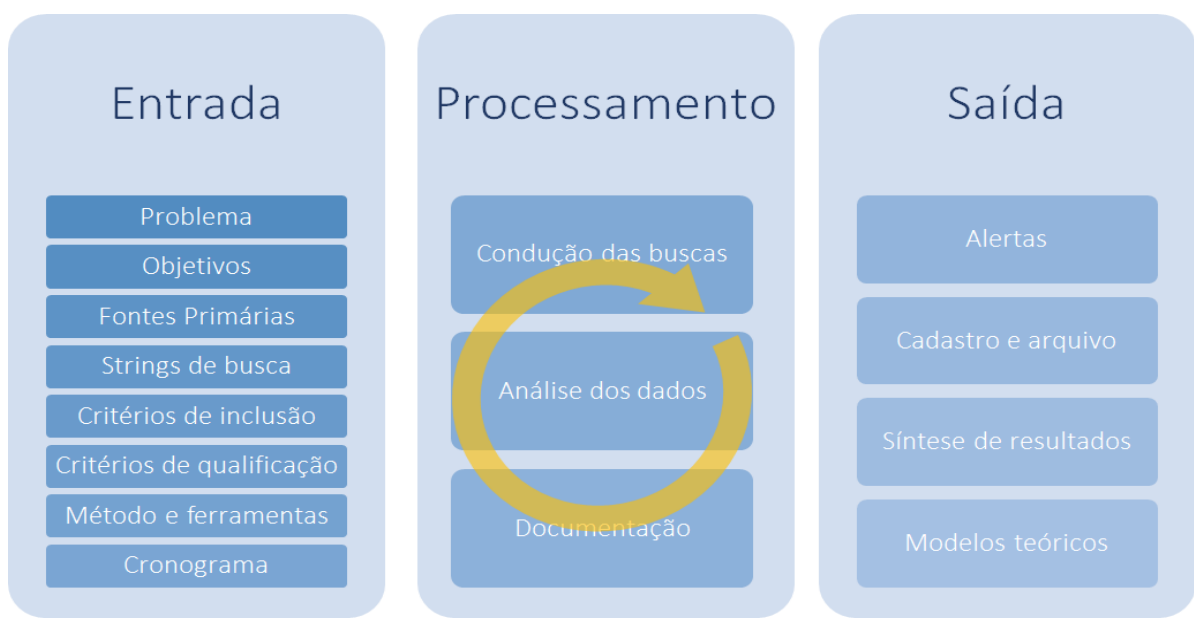

Figura 1. Processo de RSL. Adaptado de [Conforto et al., 2011].

O período de tempo das revisões sistemáticas (2008 a 2019) foi definido a partir de [Conforto et al., 2011], que sugerem a definição do recorte de um período de tempo para a realização da pesquisa e por referência aos trabalhos que foram base para a realização da revisão [Citadin et al., 2014; Cruz e Isotani, 2014; Amara et al, 2016; Maqtary et al., 2017], que em sua maioria utilizaram um período de tempo de 10 a 11 anos como recorte de tempo em suas revisões.

A questão principal da pesquisa foi definida a partir da necessidade de compreender os processos da formação dos grupos em ambientes colaborativos: 
VIII Congresso Brasileiro de Informática na Educação (CBIE 2019)

Anais do XXX Simpósio Brasileiro de Informática na Educação (SBIE 2019)

QP. Como ocorre o agrupamento de estudantes para realização de atividades que tenham como base a teoria da aprendizagem colaborativa?

E as seguintes sub questões de pesquisa foram definidas para responder a QP:

SQ1. Qual a tecnologia computacional utilizada para o agrupamento?

SQ2. Quais as características, habilidades elou categorias foram definidas para o agrupamento?

SQ3. Qual abordagem é utilizada para o agrupamento (se homogêneo, heterogêneo ou misto)?

A escolha da ferramenta de pesquisa foi o buscador Google Acadêmico, por ser uma fonte extensa para a pesquisa de estudos acadêmicos. O Google Acadêmico tem sido considerado uma fonte de pesquisa confiável, com amplo retorno de resultados em relação as bases Web of Science e Scopus, por exemplo [Martín-Martín et al., 2018].

A string de busca definida foi: ("group formation" OR "group creation" OR "team formation" OR "team creation") AND "computer supported collaborative learning" AND ("algorithms" OR "technique"). A busca inicial no Google Acadêmico resultou em 768 artigos encontrados. Foram definidos critérios de inclusão (CI) e critérios de exclusão (CE), conforme Tabela 1, para a seleção dos artigos que adequavam-se à questão de pesquisa.

Tabela 1. Critérios de Inclusão e Exclusão

\begin{tabular}{|l|l|}
\hline \multicolumn{1}{|c|}{ Critérios de Inclusão } & \multicolumn{1}{c|}{ Critérios de Exclusão } \\
\hline $\begin{array}{l}\text { CI1. Os estudos apresentam uma proposta de } \\
\text { tecnologia computacional para o agrupamento. }\end{array}$ & CE1. Não atende aos critérios de inclusão. \\
\hline $\begin{array}{l}\text { CI2. A publicação é voltada para a discussão da } \\
\text { formação de grupos para a aprendizagem } \\
\text { colaborativa. }\end{array}$ & CE2. Texto completo não disponível para acesso. \\
\hline- & $\begin{array}{l}\text { CE3. Não apresenta um estudo prático e inédito na } \\
\text { área. }\end{array}$ \\
\hline- & $\begin{array}{l}\text { CE4. Pesquisa duplicada (artigo mais recente } \\
\text { cobre as informações presentes em artigos mais } \\
\text { antigos de mesma autoria). }\end{array}$ \\
\hline
\end{tabular}

Foram definidas 3 triagens para a inclusão ou exclusão dos artigos:

$1^{\text {a }}$ triagem: seleção de todos os artigos que continham no título, resumo ou palavras-chave ao menos uma das palavras-chave "colaborativa learning" e "group formation" ou "group creation" ou "team formation" ou "team creation". Com os artigos que não cumpriam o requisito inicial era realizado a leitura de título, resumo e palavras-chave e aplicados os critérios de inclusão e exclusão. Do total dos artigos retornados pela busca, 78 artigos foram selecionados para a segunda triagem.

$2^{\mathrm{a}}$ triagem: nova leitura do título, resumo e palavras-chave e aplicação dos critérios de inclusão e exclusão. Foram excluídos da revisão os estudos em que a resposta era negativa para pelo menos um dos critérios de exclusão. Do total de 78 artigos analisados foram selecionados 52 para a terceira triagem.

$3^{\mathrm{a}}$ triagem: leitura completa dos artigos e aplicação dos critérios (CI e $\mathrm{CE}$ ) para seleção dos artigos finais. Do total de 52 foram selecionados 13 trabalhos para a extração dos dados. 
VIII Congresso Brasileiro de Informática na Educação (CBIE 2019)

Anais do XXX Simpósio Brasileiro de Informática na Educação (SBIE 2019)

\section{Resultados}

Os trabalhos selecionados são apresentados na primeira coluna da Tabela 2. Há uma grande diversidade quanto ao país de origem das pesquisas mapeadas sendo Estados Unidos e Irã com duas publicações cada e Argentina, Brasil, Espanha, Índia, Inglaterra, Quênia e Taiwan com uma publicação cada, Brasil e Colômbia com uma publicação em conjunto e Irã, Canadá e Austrália com outra publicação em conjunto.

Em relação aos anos de publicação das pesquisas, o ano com mais concentração de pesquisas é 2009, com 3 publicações, seguido de 2010, 2012 e 2014 com 2 publicações cada e 2016, 2017, 2018 e 2019 com 1 publicação cada. Considera-se que há continuidade nas pesquisas da área, sendo relevante a realização de uma RSL. Os periódicos e conferências dos artigos estão presentes na Figura 2.

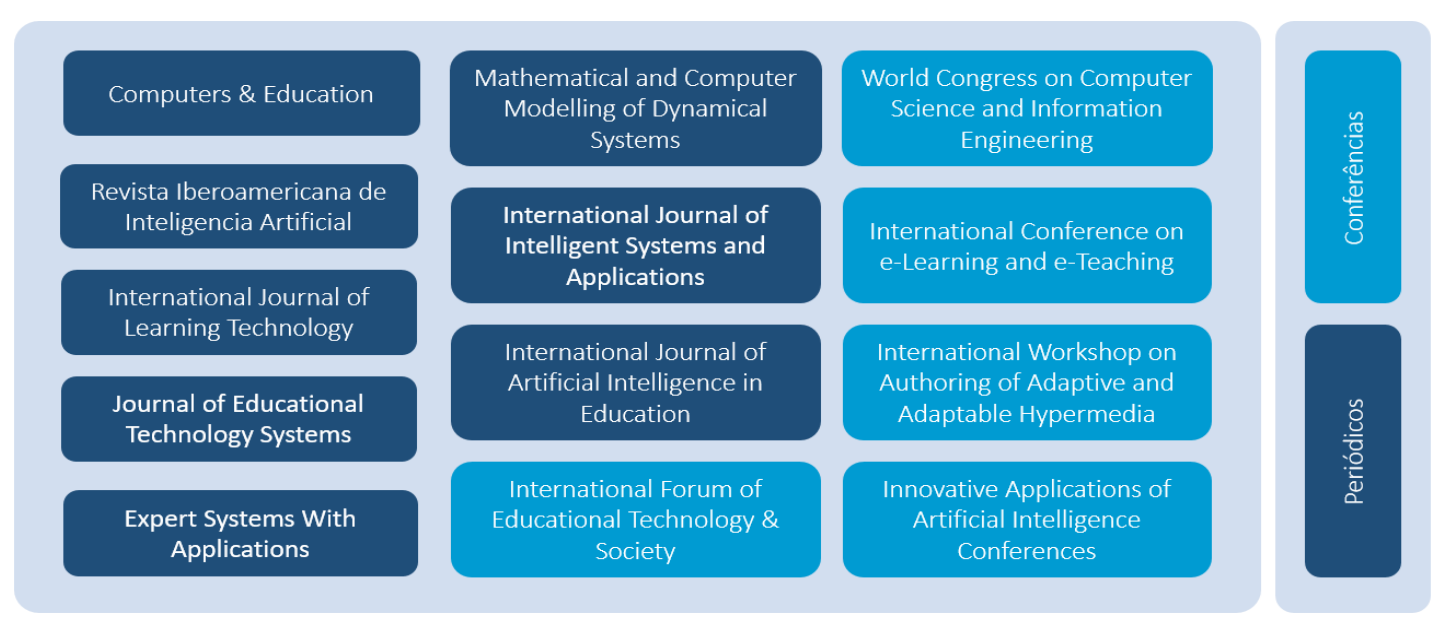

Figura 2. Periódicos e Conferências

A extração dos dados está resumida na Tabela 2, abaixo. As informações referentes as colunas 2,3 e 4 respondem as sub questões de pesquisa 1,2 e 3, respectivamente. Os dados extraídos são apresentados e discutidos a seguir.

Tabela 2. Dados extraídos

\begin{tabular}{|c|c|c|c|}
\hline $\begin{array}{c}\text { Título, autores e ano das } \\
\text { publicações }\end{array}$ & Algoritmo/método & $\begin{array}{c}\text { Características para a seleção } \\
\text { dos grupos }\end{array}$ & $\begin{array}{c}\text { Tipo de grupo } \\
\text { formado } \\
\end{array}$ \\
\hline $\begin{array}{l}\text { 1. "A genetic algorithm } \\
\text { approach for group formation } \\
\text { in collaborative learning } \\
\text { considering multiple student } \\
\text { characteristics" } \\
\text { Moreno et al. (2012) }\end{array}$ & $\begin{array}{l}\text { Algoritmos } \\
\text { genéticos. }\end{array}$ & $\begin{array}{l}\text { Nível de conhecimento; nível das } \\
\text { habilidades comunicativas; nível } \\
\text { de habilidades de liderança. }\end{array}$ & Homogêneo \\
\hline $\begin{array}{l}\text { 2. "A Framework for } \\
\text { Semantic Group Formation in } \\
\text { Education" } \\
\text { Ounnas et al. (2009) }\end{array}$ & $\begin{array}{l}\text { Ontologia; } \\
\text { problema de } \\
\text { satisfação de } \\
\text { restrições. }\end{array}$ & $\begin{array}{l}\text { Gênero; nacionalidade; idade; } \\
\text { nível de conhecimento; papéis da } \\
\text { equipe, interesses pessoais; } \\
\text { estilos de aprendizagem. }\end{array}$ & Misto \\
\hline $\begin{array}{l}\text { 3. "Composing High- } \\
\text { heterogeneous and High- } \\
\text { interaction Groups in } \\
\text { Collaborative Learning with } \\
\text { Particle Swarm Optimization" } \\
\text { Ho et al. (2009) }\end{array}$ & $\begin{array}{l}\text { Otimização por } \\
\text { enxame de } \\
\text { partículas. }\end{array}$ & $\begin{array}{l}\text { Nível de conhecimento; estilo de } \\
\text { aprendizagem; compatibilidade } \\
\text { entre colegas. }\end{array}$ & Heterogêneo \\
\hline
\end{tabular}


VIII Congresso Brasileiro de Informática na Educação (CBIE 2019)

Anais do XXX Simpósio Brasileiro de Informática na Educação (SBIE 2019)

\begin{tabular}{|c|c|c|c|}
\hline $\begin{array}{c}\text { Título, autores e ano das } \\
\text { publicações }\end{array}$ & Algoritmo/método & $\begin{array}{c}\text { Características para a seleção } \\
\text { dos grupos }\end{array}$ & $\begin{array}{l}\text { Tipo de grupo } \\
\text { formado }\end{array}$ \\
\hline $\begin{array}{l}\text { 4. "Modeling the learner } \\
\text { group formation problem in } \\
\text { computer-supported } \\
\text { collaborative learning using } \\
\text { mathematical programming" } \\
\text { Kardan e Sadeghi. (2014) }\end{array}$ & $\begin{array}{l}\text { Programação } \\
\text { binária e inteira. }\end{array}$ & $\begin{array}{l}\text { Interesses pessoais; habilidades } \\
\text { individuais }\end{array}$ & Homogêneo \\
\hline $\begin{array}{l}\text { 5. "TOGETHER: an } \\
\text { Authoring Tool for Group } \\
\text { Formation based on Learning } \\
\text { Styles" } \\
\text { Paredes et al. (2009) }\end{array}$ & $\begin{array}{l}\text { Algoritmo } \\
\text { Faraway-so-close } \\
\text { (distância } \\
\text { euclidiana). }\end{array}$ & Estilo de aprendizagem. & Heterogêneo \\
\hline $\begin{array}{l}\text { 6. "Using constraint } \\
\text { satisfaction to aid group } \\
\text { formation in CSCL" } \\
\text { Balmaceda et al. (2014) }\end{array}$ & $\begin{array}{l}\text { Problema de } \\
\text { satisfação de } \\
\text { restrições. }\end{array}$ & $\begin{array}{l}\text { Estilos psicológicos; papéis de } \\
\text { equipe; redes sociais. }\end{array}$ & Heterogêneo \\
\hline $\begin{array}{l}\text { 7. "Evaluating automatic } \\
\text { group formation mechanisms } \\
\text { to promote collaborative } \\
\text { learning - a case study" } \\
\text { Webber e Lima. (2012) }\end{array}$ & $\begin{array}{l}\text { Algoritmo de } \\
\text { agrupamento de } \\
\text { dados. }\end{array}$ & Nível de conhecimento. & Heterogêneo \\
\hline $\begin{array}{l}\text { 8. "Using Machine Learning } \\
\text { Techniques to Support Group } \\
\text { Formation in an Online } \\
\text { Collaborative Learning } \\
\text { Environment" } \\
\text { Maina et al. (2017) }\end{array}$ & $\begin{array}{l}\text { Algoritmos de } \\
\text { agrupamento de } \\
\text { dados. }\end{array}$ & Nível de conhecimento. & Heterogêneo \\
\hline $\begin{array}{l}\text { 9. "An efficacious dynamic } \\
\text { mathematical modelling } \\
\text { approach for creation of best } \\
\text { collaborative groups" } \\
\text { Kardan e Sadeghi. (2016) }\end{array}$ & $\begin{array}{l}\text { Modelo de } \\
\text { otimização } \\
\text { matemática. }\end{array}$ & $\begin{array}{l}\text { Nível de conhecimento; } \\
\text { compatibilidade entre colegas. }\end{array}$ & Heterogêneo \\
\hline $\begin{array}{l}\text { 10. "A Wiki with Multiagent } \\
\text { Tracking, Modeling, and } \\
\text { Coalition Formation" } \\
\text { Khandaker e Soh. (2010a) }\end{array}$ & $\begin{array}{l}\text { Multiagentes (a } \\
\text { partir de rede } \\
\text { bayesiana). }\end{array}$ & $\begin{array}{l}\text { Nível de conhecimento; } \\
\text { possibilidade de contribuição ao } \\
\text { grupo. }\end{array}$ & Misto \\
\hline $\begin{array}{l}\text { 11. "A “Mixed" Strategy for } \\
\text { Collaborative Group } \\
\text { Formation and Its Learning } \\
\text { Outcomes" } \\
\text { Acharya e Sinha. (2018) }\end{array}$ & $\begin{array}{l}\text { Busca dirigida por } \\
\text { agenda; algoritmo } \\
\text { de agrupamento } \\
\text { de dados. }\end{array}$ & $\begin{array}{l}\text { Estilo de aprendizagem; nível de } \\
\text { conhecimento. }\end{array}$ & Misto \\
\hline $\begin{array}{l}\text { 12. "Improving Group } \\
\text { Selection and Assessment in } \\
\text { an Asynchronous } \\
\text { Collaborative Writing } \\
\text { Application" } \\
\text { Khandaker e Soh. (2010b) }\end{array}$ & Multiagentes & $\begin{array}{l}\text { Nível de conhecimento; } \\
\text { compatibilidade entre colegas; } \\
\text { tempo gasto no sistema. }\end{array}$ & Misto \\
\hline $\begin{array}{l}\text { 13. "Optimal learning group } \\
\text { formation: A multi-objective } \\
\text { heuristic search strategy for } \\
\text { enhancing inter-group } \\
\text { homogeneity and intra-group } \\
\text { heterogeneity" } \\
\text { Garshasbi et al. (2019) }\end{array}$ & $\begin{array}{l}\text { Algoritmos } \\
\text { genéticos } \\
\text { multiobjetivos }\end{array}$ & $\begin{array}{l}\text { Gênero; nível de habilidades de } \\
\text { liderança; nível de } \\
\text { conhecimento; nível de } \\
\text { habilidades comunicativas }\end{array}$ & Misto \\
\hline
\end{tabular}


VIII Congresso Brasileiro de Informática na Educação (CBIE 2019)

Anais do XXX Simpósio Brasileiro de Informática na Educação (SBIE 2019)

\section{SQ1. Qual a tecnologia computacional utilizada para o agrupamento?}

Buscou-se identificar algoritmos e técnicas utilizados para a realização da formação de grupos. É possível notar a variedade de técnicas úteis para o agrupamento. As técnicas de computação evolutiva são utilizadas em três trabalhos mapeados [Ho et al., 2009; Moreno et al., 2012; Garshasbi et al., 2019] e são modelos baseados nos princípios da evolução de Darwin [Moreno et al., 2012], sendo estes modelos utilizados para a solução de problemas de otimização [Ho et al., 2009].

O estudo de [Ounnas et al., 2009] desenvolve uma ontologia, para formar um perfil de aprendizado dinâmico, que descreve atividades pessoais sociais e também dados acadêmicos dos estudantes, como estilos de aprendizagem e preferências. [Ounnas et al., 2009] utilizam o problema de satisfação de restrição para agrupar os estudantes, que também é utilizado no estudo de [Balmaceda et al., 2014], sendo uma restrição para que uma solução para um problema de otimização deve satisfazer [Kardan e Sadeghi, 2014].

O estudo de [Kardan e Sadeghi, 2014] utiliza a programação binária e inteira, um caso especial de programação matemática, onde função objetivo e restrições são lineares e os valores das variáveis de decisão são 0 ou 1. Outro estudo de [Kardan e Sadeghi, 2016] utiliza um modelo de otimização matemática, que consiste de uma função objetivo necessária a ser maximizada ou minimizada por meio da escolha sistemática de valores de variáveis dentro de um conjunto permitido, relacionado a possíveis restrições existentes.

Dois estudos de [Khandaker e Soh, 2010a; 2010b] utilizam sistemas multiagentes, compostos por múltiplos agentes, que interagem, mas possuem comportamento autônomo [Reis, 2003]. [Paredes et al., 2009] desenvolvem um algoritmo chamado Faraway-So-Close, um método supervisionado para a formação de grupos heterogêneos bem balanceados com base, entre outros, na distância euclidiana.

O estudo de [Acharya e Sinha, 2018] utiliza a busca dirigida por agenda, que é uma agenda que contém a lista de tarefas que um sistema pode executar, além de algoritmo de agrupamento para o agrupamento dos estudantes. Além de [Acharya e Sinha, 2018], [Webber e Lima, 2012] e [Maina et al., 2017] são outros trabalhos que utilizam algoritmos de agrupamento de dados. Algoritmos de agrupamento objetivam particionar objetos em grupos, com base em características que estes objetos possuem [Linden, 2009].

\section{SQ2. Quais as características, habilidades e/ou categorias definidas para o agrupamento?}

Conforme a Tabela 2, é possível destacar que o nível de conhecimento prévio do estudante, aquilo que ele já está familiarizado em relação ao conteúdo estudado, é a característica mais utilizada na hora de agrupar os alunos, com base nas notas anteriores, presente em 10 trabalhos da RSL [Ounnas et al., 2009; Ho et al., 2009; Khandaker e Soh, 2010a; Khandaker e Soh, 2010b; Moreno et al., 2012; Webber e Lima, 2012; Kardan e Sadeghi, 2016; Maina et al., 2017; Acharya e Sinha, 2018; Garshasbi et al., 2019].

Outras características utilizadas em mais de um trabalho foram os estilos de aprendizagem, presente em quatro trabalhos [Ounnas et al., 2009; Ho et al., 2009; 
VIII Congresso Brasileiro de Informática na Educação (CBIE 2019)

Anais do XXX Simpósio Brasileiro de Informática na Educação (SBIE 2019)

Paredes et al., 2009; Kardan e Sadeghi, 2016], estilos de aprendizagem, presente também em quatro estudos [Ounnas et al., 2009; Ho et al., 2009; Paredes et al., 2009; Kardan e Sadeghi, 2016] e a compatibilidade entre colegas de classe, presente em três trabalhos da RSL [Ho et al., 2009; Kardan e Sadeghi, 2016; Khandaker e Soh, 2010b]. níveis de habilidades comunicativas e níveis de habilidades de liderança [Moreno et al., 2012; Garshasbi et al., 2019] e gênero [Ounnas et al., 2009; Garshasbi et al., 2019]

Entre as características utilizadas apenas uma vez, cita-se: nacionalidade, idade, papéis da equipe [Ounnas et al., 2009]; habilidades individuais [Kardan e Sadeghi, 2014]; tipo de personalidade, papéis de equipe e redes sociais [Balmaceda et al., 2014]; possibilidade de contribuição ao grupo [Khandaker e Soh, 2010a]; tempo gasto no sistema [Khandaker e Soh, 2010b]. Parte das informações usadas como características são obtidas através de questionários previamente aplicados com alunos [Moreno et al., 2012; Paredes et al., 2009; Balmaceda et al., 2014; Khandaker e Soh, 2010b].

\section{SQ3. Qual abordagem é utilizada para o agrupamento?}

Em relação à estratégia estabelecida para a escolha da forma com que esse agrupamento acontecerá, três maneiras foram citadas e discutidas nos estudos da revisão sistemática: (1) Grupos heterogêneos, quando há indivíduos com características específicas distintas em um mesmo grupo; (2) grupos homogêneos, quando há indivíduos com características diferentes em um mesmo grupo; (3) e grupos mistos, quando ocorre uma combinação entre características que levam em conta a heterogeneidade e características que levam em conta a homogeneidade.

Entre as estratégias escolhidas para a composição dos grupos, 6 estudos (46\%) criam grupos heterogêneos [Ho et al., 2009; Paredes et al., 2009; Balmaceda et al., 2014; Webber e Lima, 2012; Kardan e Sadeghi, 2016; Maina et al., 2017], 5 estudos (39\%) usam o método de grupos mistos [Ounnas et al., 2009; Khandaker e Soh, 2010a; Khandaker e Soh, 2010b; Acharya e Sinha, 2018; Garshasbi et al., 2019] e uma minoria de 2 estudos (15\%) busca formar grupos homogêneos [Moreno et al., 2012; Kardan e Sadeghi, 2014], conforme a última coluna da Tabela 2.

\section{Discussão}

As informações coletadas acima são debatidas e comparadas com os estudos de [Citadin et al., 2014; Cruz e Isotani, 2014; Amara et al, 2016; Maqtary et al., 2017]. Dentre os algoritmos utilizados para realizar o agrupamento, a presente RSL encontrou uma maioria de trabalhos que utilizam algoritmos de agrupamento e algoritmos evolutivos, porém com uma grande variedade de outros algoritmos utilizados. [Maqtary et al., 2017] pontuam que em $60 \%$ dos artigos mapeados utilizam algoritmos evolutivos, a maior quantidade entre os trabalhos mapeados. Já no estudo de [Cruz e Isotani, 2014] os algoritmos mais recorrentes são os probabilísticos (41\%). Os dados extraídos de [Citadin et al., 2014] resultaram em uma quantidade mais ampla de algoritmos ou técnicas aplicadas, sendo que algoritmos evolutivos são os mais frequentes, utilizados em dois dos trabalhos mapeados cada. Já [Amara et al., 2016], que focam na aprendizagem colaborativa para dispositivos móveis, não encontraram dados suficientes quanto aos algoritmos aplicados nas formações dos grupos. Considera-se que, entre as RSL comparadas com este trabalho, há uma grande diversidade de técnicas e algoritmos aplicados para a formação dos grupos, sendo os algoritmos evolutivos o mais comum. 
VIII Congresso Brasileiro de Informática na Educação (CBIE 2019)

Anais do XXX Simpósio Brasileiro de Informática na Educação (SBIE 2019)

Em relação as características de agrupamento utilizadas, esta pesquisa encontrou uma grande maioria de estudos que utilizavam o conhecimento prévio do estudante como base para a formação dos grupos. Dentre as RSL as características que foram mais presentes foram estilos de aprendizagem [Citadin et al., 2014], experiências [Amara et al., 2016] e conhecimento prévio [Amara et al., 2016; Maqtary et al., 2017]. O estudo de [Cruz e Isotani, 2014] não contém questão de pesquisa quanto aos atributos e características dos trabalhos mapeados. Pode-se considerar que o conhecimento prévio do estudante é a característica mais levada em conta para o agrupamento dos estudantes.

Já na estratégia de agrupar os estudantes a partir das características, o presente estudo mostrou que a grande maioria dos estudos buscam agrupar de forma heterogênea os estudantes. O estudo de [Citadin et al., 2014] detecta que a grande maioria dos estudos utiliza um método misto, utilizando homogêneo e heterogêneo dependendo da característica. Já em [Amara et al., 2016] os grupos são formados em sua maioria a partir de um método homogêneo. [Cruz e Isotani, 2014] e [Maqtary et al., 2017] não trazem questões de pesquisa quanto ao método de agrupamento dos estudantes. Diferente do estudo realizado nesta RSL, os trabalhos que abordaram o método de agrupamento não encontraram uma maioria de estudos utilizando a heterogeneidade como o método mais usual. Destaca-se, porém, que o segundo método mais popular no estudo de [Citadin et al., 2014] é o heterogêneo.

\section{Considerações Finais}

A partir do debate quanto as RSL de trabalhos anteriores, foi possível realizar a comparação das sub questões de pesquisa deste estudo com os dados extraídos dos outros estudos. Com relação as técnicas utilizadas para a formação dos grupos, há uma grande diferenciação entre as RSL. Apesar das técnicas em sua maioria serem de algoritmos evolutivos [Maqtary et al., 2017; Citadin et al., 2014] e estatísticos [Cruz e Isotani, 2014], que também são detectados neste estudos, os algoritmos de agrupamento foram uma das técnicas mais recorrentes nas pesquisas na presente RSL.

Apenas duas das RSL trazem questão de pesquisa quanto ao método de agrupamento e há variedade quanto a estratégia mais usual para agrupar os estudantes, sendo misto no estudo de [Citadin et al., 2014] e homogêneo no estudo de [Amara et al., 2016]. Já o presente estudo apresenta a maioria dos trabalhos utilizando a heterogeneidade para a formação dos grupos. Em relação as características utilizadas para o agrupamento, houve consenso na maioria das RSL, sendo o conhecimento prévio do estudante a característica mais utilizada entre os estudos mapeados.

Destaca-se ainda que o volume de trabalhos mapeados neste estudo $(\mathrm{n}=768)$ foi superior as outras RSL ( $\mathrm{n}=167$ [Citadin et al., 2014], $\mathrm{n}=256$ [Cruz e Isotani, 2014], $\mathrm{n}=178$ [Amara et al, 2016] e $\mathrm{n}=110$ [Maqtary et al., 2017]), ocorrendo uma maior abrangência de estudos mapeados para a extração dos dados propostos. Esta pesquisa contribuiu com uma atualização dos dados quanto a formação de grupos para a aprendizagem colaborativa e comparação com outras RSL de objetivos semelhantes.

\section{Agradecimentos}

O presente trabalho foi realizado com apoio da Coordenação de Aperfeiçoamento de Pessoal de Nível Superior - Brasil (CAPES) - Código de Financiamento 001. 
VIII Congresso Brasileiro de Informática na Educação (CBIE 2019)

Anais do XXX Simpósio Brasileiro de Informática na Educação (SBIE 2019)

\section{Referências}

Acharya, A. e Sinha, D. (2018) A "Mixed" Strategy for Collaborative Group Formation and Its Learning Outcomes. Journal of Educational Technology Systems, v. 46, n. 4, p. $440-462$.

Amara, S., Macedo, J., Bendella, F. e Santos, A. (2016) Group Formation in Mobile Computer Supported Collaborative Learning Contexts: A Systematic Literature Review. Journal of Educational Technology \& Society, v. 19, n. 2.

Balmaceda, J. M., Schiaffino, S. N. e Pace, J. A. D. (2014) Using constraint satisfaction to aid group formation in CSCL. Inteligencia Artificial, Revista Iberoamericana de Inteligencia Artificial, v. 17, n. 53, p. 35-45.

Chen, J., Wang, M., Kirschner, P. A. e Tsai, C. C. (2018). The role of collaboration, computer use, learning environments, and supporting strategies in CSCL: A metaanalysis. Review of Educational Research, 88(6), 799-843.

Citadin, J. R., Kemczinski, A. e Matos, A. (2014) Formação de Grupos para Aprendizagem Colaborativa: Um mapeamento sistemático da literatura. XIX Conferência Internacional sobre Informática na Educação. p. 46-54.

Conforto, E. C., Amaral, D. C. e Silva, S. L. (2011) Roteiro para revisão bibliográfica sistemática: aplicação no desenvolvimento de produtos e gerenciamento de projetos. $8^{\circ}$ Congresso de Gestão de Desenvolvimento de Produto, n. 8, Porto Alegre.

Costaguta, R. (2015) Algorithms and machine learning techniques in collaborative group formation. Mexican International Conference on Artificial Intelligence. Springer, p. 249-258.

Cruz, W. M. e Isotani, S. (2014) Group formation algorithms in collaborative learning contexts: A systematic mapping of the literature. CYTED-RITOS International Workshop on Groupware. Springer, p. 199-214.

Farenhof, H. A. e Fernandes, R. F. (2016) Desmistificando a revisão de literatura como base para redação científica: método sff. Revista ACB: Biblioteconomia em Santa Catarina, v. 21, n. 3.

Garshasbi, S., Mohammadi, Y., Graf, S., Garshasbi, S. e Shen, J. (2019) Optimal learning group formation: A multi-objective heuristic search strategy for enhancing inter-group homogeneity and intra-group heterogeneity. Expert Systems with Applications, v. 118, p. 506-521.

Ho, T. F., Shyu, S. J., Wang, F. H. e Li, C. T. J. (2009) Composing high-heterogeneous and high-interaction groups in collaborative learning with particle swarm optimization. Computer Science and Information Engineering, 2009 WRI World Congress. IEEE, p. 607-611.

Kardan, A. A. e Sadeghi, H. (2014) Modeling the learner group formation problem in computer-supported collaborative learning using mathematical programming. eLearning and e-Teaching, 8th National and 5th International Conference. IEEE, p. 15. 
VIII Congresso Brasileiro de Informática na Educação (CBIE 2019)

Anais do XXX Simpósio Brasileiro de Informática na Educação (SBIE 2019)

Kardan, A. A. e Sadeghi, H. (2016) An efficacious dynamic mathematical modelling approach for creation of best collaborative groups. Mathematical and Computer Modelling of Dynamical Systems, v. 22, n. 1, p. 39-53.

Khandaker, N. e Soh, L. (2010a) A Wiki with Multiagent Tracking, Modeling, and Coalition Formation. Innovative Applications of Artificial Intelligence Conferences.

Khandaker, N. e Soh, L. (2010b) Improving group selection and assessment in an asynchronous collaborative writing application. International Journal of Artificial Intelligence in Education, v. 20, n. 3, p. 231-268.

Linden, R. (2009) Técnicas de agrupamento. Revista de Sistemas de Informação da FSMA, v. 4, p. 18-36.

Maina, E. M., Oboko, R. O. e Waiganjo, P. W. (2017) Using Machine Learning Techniques to Support Group Formation in an Online Collaborative Learning Environment. International Journal of Intelligent Systems \& Applications, v. 9, n. 3.

Maqtary, N., Mohsen, A. e Bechkowm, K. (2017) Group Formation Techniques in Computer-Supported Collaborative Learning: A Systematic Literature Review. Technology, Knowledge and Learning, p. 1-22.

Martín-Martín, A., Orduna-Malea, E., Thelwall, M. e López-Cózar, E. D. (2018) Google Scholar, Web of Science, and Scopus: A systematic comparison of citations in 252 subject categories. Journal of Informetrics, v. 12, n. 4, p. 1160-1177.

Moreno, J., Ovalle, D. A. e Vicari, R. M. (2012) A genetic algorithm approach for group formation in collaborative learning considering multiple student characteristics. Computers \& Education, v. 58, n. 1, p. 560-569.

Ounnas, A., Davis, H. C. e Millard, D. E. (2009) A framework for semantic group formation in education. Journal of Educational Technology \& Society, v. 12, n. 4, p. 43.

Paredes, P., Ortigosa, A. e Rodriguez, P. (2009) TOGETHER: An authoring tool for group formation based on learning styles. $\mathrm{A} 3 \mathrm{H}$ : 7th international workshop on authoring of adaptive and adaptable hypermedia at EC-TEL.

Reis, L. P. (2003) Coordenação em Sistemas Multi-Agente: Aplicações na Gestão Universitária e Futebol Robótico. Tese de Doutorado. Universidade do Porto.

Reis, R. C. D., Isotani, S., Rodriguez, C. L., Lyra, K. T., Jaques, P. A. e Bittencourt, I. I. (2018). Affective states in computer-supported collaborative learning: Studying the past to drive the future. Computers \& Education, 120, 29-50.

Stahl, G., Koschmann, T. D. e Suthers, D. D. (2006) Computer-supported collaborative learning, p. 409-426.

Webber, C. G. e Lima, M. F. W. P. (2012) Evaluating automatic group formation mechanisms to promote collaborative learning-a case study. International Journal of Learning Technology, v. 7, n. 3, p. 261-276. 